\title{
The voice of postsurgical lung cancer patients regarding supportive care needs
}

\author{
This article was published in the following Dove Press journal: \\ Lung Cancer:Targets and Therapy \\ 28 April 2014 \\ Number of times this article has been viewed
}

\author{
Amy J Hoffman' \\ Ruth Ann Brintnall ${ }^{2}$ \\ Alexander von $\mathrm{Eye}^{3}$ \\ Julie Cooper ${ }^{2}$ \\ Jean K Brown ${ }^{4}$ \\ 'College of Nursing, Michigan State \\ University, East Lansing, MI, USA; \\ ${ }^{2}$ Kirkhof College of Nursing, Grand \\ Valley State University, Grand Rapids, \\ MI, USA; ${ }^{3}$ Psychology Department, \\ Michigan State University, East \\ Lansing, MI, USA; ${ }^{4}$ School of Nursing, \\ State University of New York at \\ Buffalo, Buffalo, NY, USA
}

Objective: Individuals with lung cancer present with multiple comorbid conditions and complex treatment plans. They are frequently vulnerable during critical transitions in the cancer survivorship trajectory. Limited research exists on the postsurgical non-small-cell lung cancer (NSCLC) population, relative to unmet supportive care needs. However, what is known is that the lung cancer population reports significantly more unmet supportive care needs than other cancer populations. The purpose of this study was to identify the postsurgical NSCLC patients' unmet supportive care needs during transition from hospital to home and through recovery after participating in a 16-week exercise intervention.

Materials and methods: Participants were 53-73 years of age with NSCLC (stage Ib-IIIa) and participated in a 16-week light-intensity exercise program after hospital discharge. For this study, participants were interviewed 12-18 months post-thoracotomy. A qualitative design was used, incorporating a semistructured guide with open-ended questions to support discussion regarding recovery experiences through 16 weeks after transitioning from hospital to home. The interview was transcribed verbatim, and data were analyzed using content analysis. Content themes were independently coded by investigators and later combined into a single report verified through participant verification of the report.

Results: Participants reviewed and agreed with the focus group report. Dominant themes included: 1) unpreparedness for post-thoracotomy recovery; 2) significant unmet needs upon hospital discharge and throughout the cancer survivorship trajectory; 3 ) unexpected symptom burden after initial month of recovery; 4) the quality of information given when pain and fatigue were troublesome during recovery; and 5) the effectiveness of exercise during the recovery process.

Conclusion: Understanding the changing needs of this population during these transitions will assist in the development of targeted supportive care interventions, to preempt negative outcomes associated with breakdowns in care during critical transition periods of the cancer survivorship trajectory.

Keywords: quality of life, symptom, exercise, qualitative research

\section{Introduction}

Lung cancer is a serious disease classified histologically as either non-small-cell $(85 \%)$ or small cell $(15 \%)$. In particular, lung cancers are noted for their high rate of occurrence and poor prognosis worldwide, with an average 5-year survival rate of only $15 \% .{ }^{1}$ In the United States, there are currently approximately 412,226 survivors of lung cancer, with $42 \%$ having survived 5 or more years since diagnosis. ${ }^{2}$ While survival time is greater with an early-stage diagnosis, the burden associated with earlystage disease can be significant. ${ }^{3}$ Yet, little is known about patients with early-stage
Correspondence: Amy J Hoffman College of Nursing, Michigan State University, Office C246 Bott Building for Nursing Education and Research, I355 Bogue Street, East Lansing, MI 48824-13I7, USA

Tel + I 6168267820

Fax +l 6166825373

Email amy.hoffman@hc.msu.edu 
lung cancer as most investigations to date have focused on the burdens associated with advanced stage disease. ${ }^{4,5}$ Those few investigations conducted with early-stage non-small-cell lung cancer (NSCLC) patients report that patients are highly symptomatic, often with compromised functional status and quality of life. ${ }^{6-12}$ The NSCLC population is also typically older, having other comorbid conditions and undergoing complex cancer treatment plans. ${ }^{7,13}$ Not surprisingly, the limited investigations conducted show that lung cancer patients report their supportive care needs are not being met., 914 Supportive care needs are defined as "the requirement of some action or resource that is necessary, desirable, or useful to attain optimal well-being". ${ }^{15}$ Understanding the supportive care needs of patients provides an understanding of the level of need and the development of targeted interventions that may be necessary or desirable to improve the quality of care. ${ }^{16}$ While the supportive care needs of the lung cancer population are great, it is not understood what supportive care needs are unmet during the cancer survivorship trajectory. ${ }^{17}$ As a result, qualitative studies are essential, to facilitate an understanding of the changing needs that arise during the transitional stages of care for highly complex patients such as the NSCLC patient population. The purpose of this study was to identify the perceptions of postsurgical NSCLC patients, relative to their unmet supportive care needs during critical transitions from hospital to home and subsequently, during recovery and participation in a 16-week rehabilitative exercise program. ${ }^{18}$

A growing body of evidence shows that adults with multiple comorbid conditions and complex treatment plans, such as those with NSCLC, are vulnerable during critical transition points along the cancer survivorship trajectory. ${ }^{19,20}$ This is particularly true for postsurgical patients during their transition from hospital to home. ${ }^{21,22}$ Likewise, the Institute of Medicine ${ }^{23}$ strongly recommends that providers anticipate rather than react to common types of patient needs. The Institute of Medicine, ${ }^{24}$ and Wen and Gustafson ${ }^{25}$ underscore the significant value of needs-based research by stating that having a more complete understanding of a patient population allows for enhanced prioritization of resources to optimize quality of life. This study aimed to uncover an in-depth understanding of the highest priority unmet supportive care needs of post-thoracotomy NSCLC patients, using an established and accepted focus group methodology. This methodology facilitates the sharing of complex experiences, to obtain targeted qualitative information from a hard-to-reach group and gain their perspective on the lived experience. ${ }^{26,27}$

\section{Methods \\ Design, setting, and participants}

This qualitative study using a focus group methodology was approved by the Institutional Review Boards at Michigan State University, Grand Valley State University, and an affiliated university teaching hospital in Michigan. Using a telephone script approved by the Institutional Review Boards, the first author extended an invitation to participate in the focus group to seven post-thoracotomy NSCLC individuals. These individuals participated in a 16-week light-intensity exercise program using the Nintendo Wii Fit Plus (Nintendo of America, Redmond, WA, USA) after hospital discharge ${ }^{18}$ and were interviewed from 8 to 14 months postexercise intervention. Of the seven who responded, six consented to participate in the focus group. One individual was unable to participate in the focus group, due to an extended out-of-the-area family event. Eligibility criteria to participate in the 16-week light-intensity exercise program and subsequently, the focus group included: 1) being 21 years of age or older; 2) undergoing thoracotomy with post-thoracotomy confirmation of NSCLC; 3) medical clearance to participate in the exercise program; 4) having stable comorbid conditions; and 5) having a Karnofsky performance score of $70 \%$ or greater (functioning at a minimum of being able to live at home and caring for most personal needs but limited in carrying out normal work activity). Participants were excluded if they had severe impairments in vision, hearing, or speaking, weighed more than 300 pounds, had a history of dementia or seizures, or had any additional conditions inhibiting safe utilization of the exercise equipment and participation in the exercise program.

\section{Study procedures}

Using guidelines by Krueger and Casey, ${ }^{27}$ the focus group was held in a private conference room at a centrally located participating university, with convenient parking. Written consent was obtained for participation and audio recording, prior to the start of the focus group by, two nationally certified oncology nurses. The first and second authors (investigators) cofacilitated the 2-hour focus group session. The investigators began with group-forming activities, including reviewing the informed consent, the purpose and format of the focus group discussion, the focus group main questions, and selfintroductions. The investigators reminded participants to respect the privacy of others and not to repeat what was said in the focus group to others. Participants received a $\$ 50$ gift card at the end of the focus group, in appreciation for time and willingness to share experiences and to compensate them for travel to the focus group session. 


\section{Data collection}

Participants' experiences pre- and post-thoracotomy were gathered using a semistructured guide with open-ended questions. Using a directed approach to content analysis, ${ }^{28}$ the semistructured guide was developed by the research team and was based on a synthesized integrated model of the Theory of Symptom Self-Management ${ }^{29,30}$ and the Transitional Care Model..$^{20,31}$ The focus group guide (Table 1) consisted of questions with probes designed to direct attention to the most salient times where gaps in care may have occurred, resulting in unmet supportive care needs. The investigators monitored the time, used the focus group guide to stimulate discussion, and ensured all participants had the opportunity to share their experiences. About 15 minutes were allotted for each main question, unless saturation was reached earlier (eg, we began to hear repeating comments). Participants were provided a 10-minute break halfway through the session. The focus group session was audio-recorded, professionally transcribed verbatim, and reviewed for accuracy by the investigators.

Table I Interview guide

Question I: What were your recovery expectations prior to surgery, and did your recovery expectations change upon returning home from the hospital?

Probes:

- How did your recovery expectations change upon learning you needed chemotherapy and/or radiation treatment?

- How did your recovery expectations change while receiving chemotherapy and/or radiation treatment?

- How did your recovery expectations change at other points during your recovery process?

- How were you able to achieve your recovery expectations?

- How did participating in an exercise program using the Wii ${ }^{\mathrm{TM}}$ have an impact on your recovery expectations?

Question 2: After discharge from the hospital, what were your most significant met/unmet needs at this time when you arrived home?

Probes:

- Particular needs in the first 72 hours.

- Particular needs in the first month.

Question 3: For those of you who required chemotherapy and/or radiation therapy, thinking back, what were your most significant met/unmet needs at this time?

Probes:

- Immediately prior to starting chemotherapy and/or radiation therapy.

- During chemotherapy and/or radiation therapy.

- What are your most significant unmet needs at this time, and where are you in the recovery process?

Question 4: For those of you who did not have additional treatments, what were your most significant ongoing met/unmet needs during your recovery? Probes:

- Thinking in particular to your activities of daily living.

- Are there other important times that we have not talked about?

- What are your most significant unmet needs at this time, and where are you in the recovery process?

Question 5: Can you tell me what your top three most troublesome symptoms have been since your diagnosis and rank the symptoms in order of significance, starting with the most troublesome symptom?

Probes:

- Tell me how the symptoms interfered with your life.

- Tell me about any other troublesome symptoms that were persistent?

Question 6: Relative to pain, after returning home from the hospital, was there a specific time period during your recovery where pain was particularly troublesome?

Probes:

- Tell me more about each pain location and the origin of the pain.

- What was not helpful in obtaining pain relief?

- Are there other things you want to tell us about your pain?

Question 7: Relative to fatigue, after returning home from the hospital, was there a specific time period during your recovery where fatigue was particularly troublesome?

Probes:

- What was not helpful in obtaining fatigue relief?

- Are there other things you want to tell us about your fatigue?

Question 8: With regard to exercise, are you continuing to exercise since leaving the Wii program?

Probes:

- If yes, what exercise are you doing; how frequently per week; and for how many minutes per day?

- Tell me anything that could have been added to the exercise program that would have been helpful?

- Tell me about any barriers you are facing to exercise.

- What are you doing to stay well? 


\section{Data analysis}

Qualitative content analysis was used to analyze the data. This approach allowed the data to be systematically classified into themes, after repeatedly reading the transcribed text to achieve a full awareness of patients' perceptions. ${ }^{27,28} \mathrm{Next}$, the data were coded, making notes about initial impressions, thoughts, and analyses, and then, sorting the codes into meaningful themes in accordance with the questions in the semistructured guide. Anonymous participant quotes were used as rationale to support the themes. Reliability of the results was addressed through high-quality audio recording and transcription of the recording. ${ }^{26}$ To maximize the credibility of the results, the two investigators and a doctoral student initially worked on the transcription independently and later, cross checked for similarities and differences while discussing the findings, to reach consensus on the joint analysis. ${ }^{26,27}$ Differences in the independent analyses were rare and were addressed by the two investigators and the doctoral student as a group, such that the differences were first compared with the transcript and then discussed as a group until consensus was reached. Further verification of the joint analysis included asking each participant to review and comment on a report that captured the theme from each of the main questions asked in the focus group ${ }^{27}$ This report was mailed to the participant's home, with a return addressed envelope for participants to mail back their input to the investigators. The participants were able to review and provide any further comments to the report by indicating agreement, partial disagreement, and/or disagreement with the summarized report, and a space was provided to include further comments. The investigators and doctoral student reviewed and incorporated the participants' responses to the report. ${ }^{27}$

\section{Results}

\section{Participant characteristics}

Six individuals, with an age range of 53-73 years at the time of surgery for NSCLC, participated in the focus group. All had a lobectomy for NSCLC. Stage of cancer ranged from Ib to IIIa. Participants were Caucasian, with a range of
2-12 comorbid conditions prethoracotomy. Table 2 displays further descriptive characteristics of the participants.

\section{Themes}

All six participants returned the report, indicating complete agreement with the themes captured from each of the main questions by the research team, and none provided any further comments. Since significant overlap in participant responses was found between questions \#3 and \#4, the summaries of the two main questions were combined. The following section describes the themes, with supporting quotations that further illustrate the participants' feedback.

\section{Theme I: unpreparedness for recovery process}

When asked about their post-thoracotomy recovery expectations, the group's expectations varied. For example, some participants did not realize how extensive the surgery really was, with one participant stating:

I just figured it was going to be an operation. I'll be better in 6 weeks. I found out that that's not true at all. In fact, I still have pain. I'd had many surgeries in the last couple - few years. And so I just figured I was going to treat the lung surgery like everything else. I was going to get home, and I was going to start moving, and things were going to go, you know, back to normal. [ID4]

Some participants found it more difficult to recover and return to their normal activities of daily living once they returned home from the hospital, with another commenting that:

I was always the caregiver, the one on the other side of the fence. And so this was just like a slap in the face like from beyond. And I was so blown away that this was happening to me. [ID5]

Likewise, all five of the participants, who were employed, relayed that they were unprepared for the impact their

Table 2 Patient characteristics

\begin{tabular}{lllllll}
\hline ID number & Sex & Age (years) & $\begin{array}{l}\text { Stage of } \\
\text { cancer }\end{array}$ & $\begin{array}{l}\text { Number of } \\
\text { comorbid conditions }\end{array}$ & $\begin{array}{l}\text { Employment } \\
\text { status }\end{array}$ & $\begin{array}{l}\text { Adjuvant treatment } \\
\text { following thoracotomy }\end{array}$ \\
\hline I & F & 64 & IIIA & 5 & Employed & Chemotherapy with radiation \\
2 & M & 73 & IIB & 6 & Employed & Chemotherapy \\
3 & F & 61 & IIB & 12 & Homemaker & Chemotherapy with radiation \\
4 & F & 66 & IB & 7 & Employed & None \\
5 & F & 53 & IIIA & 2 & Employed & Chemotherapy with radiation \\
6 & M & 66 & IIA & 2 & Employed & Chemotherapy \\
\hline
\end{tabular}


recovery would have on their work. Each expressed varying issues in trying to return to work and concern over the financial impact this would have, with one participant stating:

I couldn't do my job after my surgery because my job is physical - so that [not being able to do my job] was worrisome, and that's fatiguing to me. [ID5]

Another stated:

I found out that I could work on the computer, and my fingers would not do on the keyboard what my mind was telling it to do. I never wanted to retire, but it's becoming more and more of an option. [ID2]

One participant also described financial hardship associated with the treatment of the disease and the distress experienced by the following event as it unfolded:

My prechemo nurse sat me down and showed me the drugs I was going to be taking, and then they sent in the financial person - they wanted to know how I was going to pay for my chemo. [ID5]

As far as recovery process expectations, some had no expectations for what would happen in recovery, with one who said:

In terms of expectations, I had none. I had no idea what I was getting myself in for. [ID6]

Others had expectations that proved false as the recovery was more difficult than anticipated. Another described a "cancer shadow" (ID2) and portrayed the experience with lung cancer as different from other illnesses. The participants realized that lung cancer is especially serious and may require various modes of treatment and follow-up. All recalled the date and time of diagnosis and surgery, which suggests that diagnosis and surgery were significant events in their lives.

\section{Theme 2: most significant unmet needs after hospital to home transition - first month}

All participants described unmet symptom-management needs after hospital discharge, with pain and shortness of breath as the most troublesome symptoms. Overall, the participants reported that their pain was not well-controlled and that their efforts at managing pain were ineffective much of the time. Two participants characterized it as follows:

I didn't realize how much pain that was going to be (the thoracotomy) - the pain in the ribs. [ID6]
I still have the same pain, and as far as I'm concerned, they have never met my pain [management expectations], even with the exercise and stuff. I still have it, and I still go around at night holding my side because it hurts so much. [ID4]

In regards to pain management, four out of six participants voiced concerns about being perceived as "addicts", "abusers", or "users". (IDs 1, 3, 4, and 5) There were also concerns of being regarded as "a bother" by health care providers, after calling the providers to report ineffective pain relief. One participant described their experience as follows:

I'm thinking, I got to get off these pills. I got to quit taking all this medicine. And you know, I tried to go longer, and I couldn't.... I'm trying to make them last longer because I didn't want to call the doctor and say, "I need pain meds" because I didn't want him to think I was turning into an addict. Yeah, and it's like “Oh, she's abusing her pain meds.” [ID5]

At times, most participants' shortness of breath limited what they could do, and participants provided a description of what they experienced in vivid, somewhat disturbing terminology, with one participant saying:

I could not [breathe] - my lung was like I had no lung at all. It would shrink down. I couldn't breathe outside. If I would just go outside, even if I were going to go to the mailbox, I was gasping for air. [ID3]

Three participants continued to describe their experiences with the symptom of shortness of breath as follows:

It's like trying to breathe underwater. It's like you're breathing, but it's not helping. [ID1]

It's shortness of breath, and it's an odd, heavy, hunger or deprivation. [ID3]

You're trying to breathe deeper, and it's not letting you. [ID5]

Several of the participants described ways they worked around their shortness of breath. Participants also reported flare-ups of other chronic illnesses that were made worse by their pain. At times, many participants felt a lack of communication with health care providers that persisted as they tried to manage cancer, symptoms, and other illnesses.

\section{Theme 3: unexpected symptom burden - after initial month of recovery}

Many of the most significant unmet needs reported by participants centered on their unexpected symptom burden and 
the lack of symptom-management strategies. All participants felt that they were not prepared nor told how to manage their symptoms and that there was a lack of information relative to symptom management and what to expect. For example, participants experienced ongoing symptoms, such as shortness of breath, and at times, did not know what to do or whether to push themselves to complete even activities of daily living. One participant stated the following:

When I took my first shower at home, and when you let the water run over your head to wash your hair, keeping your mouth shut, it was like a panicky feeling because it was like I had to hurry up and get out of the water so I could open my mouth and breathe, and I couldn't do that for very long, only for a few seconds but that was scary. That was frightening to me when I first got home because nobody told me, I mean, who would have thought? [ID5]

For those who received certain chemotherapy and/or radiation, pain was common and persistent and was often worse for those who received both. For instance, one participant described the pain as follows:

One of the things that I had a lot of from chemo is bone pain and joint pain, and I still have a lot of that, especially in my legs. [ID5]

Participants also described psychological symptoms and the stress of returning home from the hospital, with one expressing this feeling in the following way:

One of the unmet needs that I didn't realize I needed was the emotional aspect of going home and trying to cope with the family, and I had a real hard time. And finally, they called the doctor and put me on some emotion pills and something to calm me down. [ID2]

Other participants reported cognitive symptoms after chemotherapy, with one participant describing this difficulty as follows:

There are days when someone will ask you a question in the store, and you know, you're just like an idiot because you can't even form a full sentence. They just look at you like, “okay. ...” [ID3]

Overall, participants described a sense of loss and frustration at being provided with conflicting expectations, with one participant tearfully recalling the loss of her hair:

I lost all my hair, and I had a couple nurses tell me I wouldn't lose it, and then I had nurses tell me I would.
So I really didn't know what was going to happen but, probably after my second treatment of chemo, it was coming out in clumps, and I couldn't stand it. It was in the sink. It was everywhere. [ID5]

Participants said another unmet need was the uncertainty they had about how their chemotherapy was going to be administered and its corresponding side effects. One participant summed up the experience as follows:

The nurse educator didn't tell me how much pain the Navelbine was going to cause when they put it in my vein. The cisplatin didn't bother, but the Navelbine was terrible. I went back to taking Vicodin for the pain in my arm. By the second treatment, I had the port put in because my chemo nurse said, "You're not going through that again." [ID6]

Another participant said with distress:

I'm on my third type of chemo and no port yet, but there are days when I get stabbed with the IV needle four to six times before they catch a vein. [ID1]

One observation that was striking was the impression left on the participants when they went for their first chemotherapy treatment and were unprepared for what they saw. Their comments were as follows:

I was overwhelmed by the magnitude of the people getting chemo - all the hours of the day, and they're full of people with cancer. [ID3]

It's "wait in line for a chair" ... and everyone is bald, and everybody is thin and looks decrepit ... it's scary it's like is this where I'm headed? [ID5]

I thought I was bad, and then I went in there, you know, for the first treatment, and I said to my spouse, I said, "My God," some of these poor people are just, you know - I never thought. [ID6]

But for the grace of God ... [ID1]

\section{Theme 4: time where pain was most troublesome - throughout recovery process}

Pain was often a troublesome symptom for many participants. While some reported improvement in surgical pain as they healed, others did not. Often new symptoms of pain began when additional treatment was started. One participant described this as follows:

Right when you're starting to get your pain under control, then they slapped me with chemo, and then it's a whole 
new ball game. It's a whole new kind of pain on top of your other pain. [ID5]

Two participants discussed the esophageal pain they experienced during their radiation treatments, and several reported having difficulty with eating and swallowing due to this, as this participant explained:

They told me that it would affect the esophagus and that swallowing would become difficult. That is putting it mildly. [ID1]

They radiated my chest, and I had a lot of esophageal pain, and I couldn't eat any solid foods. [ID5]

\section{Theme 5: time when fatigue was most troublesome - throughout recovery process}

Participants described cancer-related fatigue as different from that of normal fatigue, as described by one participant:

Cancer fatigue I found to be a lot different than physical fatigue. Physical fatigue you can sit down and take a nap. I can't do that with the cancer fatigue. [ID2]

In terms of timing, one participant was surprised by the intensity of fatigue after hospitalization stating:

My fatigue ... was basically when I got home because the surgery had just taken so much.... I wasn't prepared for what it all was going to be, and I was in the hospital for nine days so ... my fatigue was worse then [on return home]. [ID6]

Participants also described how fatigue shared a characteristic pattern with pain in that once participants got their strength back, chemotherapy was introduced, and fatigue came back again and/or intensified. The fatigue experienced during the surgical recovery period was described differently from the fatigue experienced when undergoing adjuvant treatment with either chemotherapy and/or radiation. Fatigue during surgical recovery alone was described as more physical and was in a localized area. Participants reported the profound severity and interfering nature of fatigue in combination with chemotherapy and/or radiation to be like:

... somebody pulled a curtain over you and falling in a bottomless hole. [ID3]

Others remarked how undergoing chemotherapy treatmentinduced fatigue since the therapy could last 6 to 7 hours per day. Others remarked how the outpatient chemotherapy clinic environment, referred to as the "combine", "is enough to wear anybody out" [ID5]. Lastly, participants also expressed how living under the shadow of being a cancer survivor was fatiguing in itself; one participant expressed as follows:

Cancer puts a weight on you, and having that weight, that load, is fatiguing. I mean okay, my CT [computed tomography] scans are clean, but every time it gets close to the next one, you get that weight again on your shoulders of worrying ... what if it's not clean? I got to go back on chemo. I got to lose my hair ... so all the worries - the worrisome of it $\ldots$ and that's fatiguing to me. [ID5]

\section{Theme 6: effect of having a rehabilitative exercise program}

One supportive care need that participants described as being met was being provided some control over their rehabilitation through their Wii exercise program, with one participant stating:

The Wii is - is a fantastic machine. I'm still using it, and I have almost 213 hours on it now. I look forward to using it in the morning because it gets endorphins in the brain going, and it starts me for the rest of the day. [ID2]

This sentiment was echoed by the other participants, with several participants making supportive statements, such as:

I do think the Wii is an excellent idea. But I think it should be expanded to more than just lung surgery. I think anybody that has had major surgery needs to get off of their butt. [ID1]

The Wii makes you feel good because you can do it. [ID5]

Many participants continued using the Wii and other forms of exercise after the intervention was over, such as joining the gym, biking, camping, walking their dog, boating, and even horseback riding. In fact, some considered the Wii exercise program and the corresponding nurse visits as the best thing that ever happened to them (IDs 1, 2, 5). Participants liked their exercise-intervention data collectors and felt it was important to have someone who cared about them call on a weekly basis to record their symptoms, exercise, and the progress the participants made. Participants also liked the paperwork, which allowed them to see their progress, including the minutes of exercise, their heart rate during exercise, and the steps per day measured with their pedometer. Last, as some participants began to feel better and with increased confidence in the Wii exercise program (around the fourth week of exercise), they wanted to expand beyond the Wii 
exercise and commence other forms of exercise that were of interest to them.

\section{Summary of themes}

It was clear from the discussion that participants were not prepared for how extensive a surgery they were facing with a thoracotomy and that they did not have an appreciation for what they would be going through during recovery. Pain, fatigue, and shortness of breath were significant symptoms reported by all, with chemotherapy and radiation introducing significant additional symptom burden. All participants appreciated having a rehabilitation program that gave them hope and a means of addressing their fatigue during recovery.

\section{Discussion}

In 2005, the Institute of Medicine report "From Cancer Patient to Cancer Survivor: Lost in Transition" noted that cancer survivorship starts at the point of a patient's diagnosis and continues through the balance of his or her life. ${ }^{24}$ The report also stated that the goal throughout survivorship is to improve the health and the quality of life for those with a history of cancer. ${ }^{24}$ This focus group consisted of six participants who were 12 to 18 months post-thoracotomy for lung cancer, who described their survivorship journey as laden with difficulties and unmet supportive care needs. Further, to the best of our knowledge, this is the first study to document the post-thoracotomy patient report of unmet supportive care needs experienced throughout the recovery process, from discharge from the hospital through 12 to 18 months postdischarge. The multiple detailed unmet supportive care needs identified in this study are best described when grouped into three fundamental categories that together, provide valuable insight crucial to the mission of improving survivorship care in individuals with lung cancer.

\section{Participant unpreparedness for their post-thoracotomy recovery}

First, participants reported being unprepared and not knowing what to expect throughout their post-thoracotomy recovery. This is consistent with previous literature reporting that among the cancer populations, patients are dissatisfied with the amount and type of information they receive about their diagnosis, cancer treatment, and ways to manage the effects of cancer and its treatment. ${ }^{23,32}$ For example, our focus group participants described the importance of knowing specific details relative to their lung cancer care, such as wanting to know what they were going to feel like after having a thora- cotomy and what to expect when they returned home from the hospital after thoracotomy. Participants also expressed that they had no idea what to expect regarding how their dayto-day activities would be affected. Participants reflected on how everyday activities, such as taking a shower and washing their hair, became scary events because of the difficulty they had taking a breath. Furthermore, others were given conflicting information on whether or not they would lose their hair during chemotherapy. In addition, consistent with the literature, the participants in this focus group frequently described situations using words that characterized significant psychosocial distress.

Studies have shown that oncology health care providers may have underestimated the level of psychosocial distress experienced by their patients..$^{3,8-10,33-35}$ In addition, it has been shown that oncology health care providers failed to screen and/or address identified psychosocial needs of their patients. ${ }^{32}$ If left unaddressed, being unprepared with incomplete information can adversely affect other areas of the health and health care of cancer survivors. For example, participants expressed fear of not fully understanding the length of their treatment and likelihood of making a full recovery, revealing concerns about financial hardship if not being able to return to work in a timely fashion. Participants shared the struggles of what this meant in terms of not having an adequate income to buy the everyday basics in life, such as food and transportation, with the new, competing needs for life-saving cancer care, such as medication or adjuvant chemotherapy. Unfortunately, research has shown a clear link between psychosocial distress and greater functional impairments. ${ }^{4}$ Consequently, a patient-centered plan of care needs to be developed between health care providers and the patient, to address this critical unmet need.

The Institute of Medicine recommends an oncology health care delivery system be equipped to provide care that is patient-centered, defined as "providing care that is respectful of and responsive to individual patient preferences, needs, and values and ensuring that patient values guide all clinical decisions." ${ }^{23}$ Patient-centered care includes fostering good communication of information, in ways that are understandable by the patient. This is important, to promote active self-management participation of the patient in their care and in turn, promote health and a high quality of life. At the most fundamental level, patients and their family members need understandable evidenced-based information that can help to establish a plan of care, with information regarding their cancer and prognosis, treatment options, the treatment's effect on their health, the total cost of cancer care, and the 
resources available to support patients to achieve quality cancer care. ${ }^{23}$ While developing a plan of care is critical, incorporating symptom-management strategies in the plan, to promote active symptom self-management, is essential.

\section{Participant unpreparedness for symptom management}

A supportive care need that is most basic and essential to cancer survivorship that was overwhelmingly reported as being unmet (except in the area of fatigue, where participants reported the exercise intervention met this need) was symptom management. Unfortunately, existing research has documented inadequate symptom management regardless of the type of cancer or of where a patient is in the cancer survivorship trajectory. ${ }^{36}$ Similarly, the most recent guidelines from the American College of Chest Physicians emphasized that the most difficult aspect of managing patients with lung cancer can often be the symptoms they experience. ${ }^{37}$ In fact, as compared with other cancer diagnoses, individuals with lung cancer reported higher levels of symptom burden. ${ }^{36}$ The participants in our study gave particular mention to the issues they encountered in managing the common symptoms of pain, dyspnea, and psychological distress. Many participants said they did not take their pain medication as prescribed because they were afraid they would run out. Likewise, they were afraid to call their health care provider for more pain medication if they ran out because they were worried they would be labeled "as an addict". These same participants also described how their pain exacerbated their other symptoms, prolonging their recovery. The participants did acknowledge that the medication, when taken, did a good job of relieving their pain; however, they were not given enough to cover their recovery period, causing them to create their own pain management regimen that was not effective.

Likewise, participants reported difficulty in managing their dyspnea. Participants said they were not prepared for what the dyspnea would be like post-thoracotomy and were not given any specific management strategies. Participants reported that the dyspnea created fear and panic when they had difficulty breathing during a shower or during everyday tasks. Since they did not know what to expect and were given no dyspnea management strategies, they discovered the effects of this disturbing symptom on their own, with nothing available to them to address it. The prevalence of these symptoms in post-thoracotomy patients suggests patients need education to learn symptom self-management, to help them address the symptoms they can expect to experience during their recovery.

In this study, participants were provided with a fatigue self-management light-intensity exercise program to help them manage their fatigue. While participants said they experienced cancer-related fatigue, all were glad they had education on what to expect with their fatigue, and all were very encouraged that they had a means to address their fatigue. Expanding the fatigue-management program to include other symptoms, such as pain, dyspnea, and other common troublesome symptoms associated with lung cancer and its treatment, would provide an encompassing, patient-centered recovery plan to address the unmet supportive care needs of symptom management.

\section{Increased attention to creating a healthy lifestyle through health promotion}

Last, the ultimate goal in cancer survivorship is to optimize quality of life, and one way to do so is by providing patients with empowering learning opportunities to change to healthy lifestyle practices. With this in mind, the focus group participants were asked to identify their most significant unmet needs during recovery from thoracotomy for NSCLC and to report on those needs that were met as well. Except for the light-intensity exercise program to promote fatigue management and improve functional status, participants did not report any area of health promotion or disease prevention offered to them that promoted their recovery from lung cancer surgery. At the same time, the postrecovery period is an opportune moment to provide patients with rich resources for a healthy lifestyle as well as control, leading to optimal health outcomes. Growing evidence shows that the adoption of healthy behaviors may also benefit survivors in that they provide improved symptom management, leading to enhanced functional status and quality of life..$^{38}$ Participants of this focus group remarked that exercise helped them gain control over their rehabilitation and allowed them to return to everyday activities. Research has shown that after receiving a life-threatening diagnosis, survivors were more motivated to make healthy lifestyle choices. ${ }^{39}$ Participants in this focus group remarked that the opportunity to join an exercise program to promote fatigue management and improve their physical functioning motivated them to initiate their own exercise program, which led all six participants to adopt lifetime exercise routines.

\section{Limitations}

This manuscript reflected the experience of six post-thoracotomy patients who completed a rehabilitation program for 
fatigue management. All participants were Caucasian, and all had surgery in the same hospital. Future studies could investigate whether these same issues are experienced by a more diverse patient population having surgery in differing hospitals and not having participated in an exercise program after surgery.

\section{Conclusion}

This focus group of six survivors of early-stage NSCLC contributed their experiences from a 12- to 18-month period of time during which they were recovering from surgery for their lung cancer. The survivors identified major deficiencies and labeled these deficiencies as significant unmet supportive care needs. Recurring and unmet supportive care needs included a lack of information that could have prepared participants to be confident and active participants in their own cancer care and treatment. Moreover, feeling unprepared contributed to their psychosocial distress and their inability to self-manage cancer symptoms. The most significant cancerrelated symptoms identified by the focus group participants included pain, fatigue, dyspnea, and psychosocial distress. These are common symptoms that if left unmanaged, lead to undue suffering and diminished quality of life. ${ }^{36,40}$ While all six participants reported receiving support through their participation in a research study, no other supportive health promoting resources were provided to this group. Lung cancer, when compared with other types of cancer, is known for greater symptom burden, with greater unmet supportive care needs. ${ }^{14,41}$ While one would think that the greatest focus would have been on support for those burdens, this was not true for the six participants in this study. In 2006, the Institute of Medicine reminded all health care providers, in their report, that:

The challenge in overcoming cancer is not only to find therapies that will prevent or arrest the disease quickly, but also to map the middle ground of survivorship and minimize medical and social hazards. ${ }^{24}$

Since then, several important reports and guidelines have provided strong recommendations by expert committees, that can be leveraged to improve care. ${ }^{23,32,38}$ Incorporating these initiatives into a plan of care for post-thoracotomy patients while continuing to learn from post-thoracotomy survivors will help to develop an effective means of equipping postthoracotomy patients with a patient-centered recovery plan and an environment that meets all of their most significant supportive care needs.

\section{Acknowledgment}

Funding for this research was received from the Michigan State University College of Nursing, East Lansing, Michigan; and the Sigma Theta Tau International Society of Nursing, Alpha Psi Sigma Theta Tau Chapter.

\section{Disclosure}

The authors report no conflicts of interest in this work.

\section{References}

1. American Cancer Society. Cancer Facts and Figures 2013. Atlanta, GA: American Cancer Society; 2013. Available from: http://www. cancer.org/acs/groups/content/@epidemiologysurveilance/documents/ document/acspc-036845.pdf. Accessed October 31, 2013.

2. de Moor JS, Mariotto AB, Parry C, et al. Cancer survivors in the United States: prevalence across the survivorship trajectory and implications for care. Cancer Epidemiol Biomarkers Prev. 2013;22(4):561-570.

3. Lowery AE, Krebs P, Coups EJ, et al. Impact of symptom burden in post-surgical non-small cell lung cancer survivors. Support Care Cancer. 2014;22(1):173-180.

4. Maliski SL, Sarna L, Evangelista L, Padilla G. The aftermath of lung cancer: balancing the good and bad. Cancer Nurs. 2003;26(3): 237-244.

5. Maguire R, Kotronoulas G, Papadopoulou C, Simpson MF, McPhelim J, Irvine L. Patient-reported outcome measures for the identification of supportive care needs in people with lung cancer: are we there yet? Cancer Nurs. 2013;36(4):E1-E17.

6. Brown JK, Cooley ME, Chernecky C, Sarna L. A symptom cluster and sentinel symptom experienced by women with lung cancer. Oncol Nurs Forum. 2011;38(6):E425-E435.

7. Yang P, Cheville AL, Wampfler JA, et al. Quality of life and symptom burden among long-term lung cancer survivors. J Thorac Oncol. 2012;7(1):64-70.

8. Sarna L, Cooley ME, Brown JK, Chernecky C, Elashoff D, Kotlerman J. Symptom severity 1 to 4 months after thoracotomy for lung cancer. $\mathrm{Am}$ J Crit Care. 2008;17(5):455-467; quiz 468.

9. Sanders SL, Bantum EQ, Owen JE, Thornton AA, Stanton AL. Supportive care needs in patients with lung cancer. Psychooncology. 2010;19(5):480-489

10. Handy JR, Asaph JW, Skokan L, et al. What happens to patients undergoing lung cancer surgery? Outcomes and quality of life before and after surgery. Chest. 2002;122(1):21-30.

11. Möller A, Sartipy U. Associations between changes in quality of life and survival after lung cancer surgery. J Thorac Oncol. 2012;7(1): 183-187.

12. Schulte T, Schniewind B, Walter J, Dohrmann P, Küchler T, Kurdow R. Age-related impairment of quality of life after lung resection for nonsmall cell lung cancer. Lung Cancer. 2010;68(1):115-120.

13. Given CW, Given B, Azzouz F, Kozachik S, Stommel M. Predictors of pain and fatigue in the year following diagnosis among elderly cancer patients. J Pain Symptom Manage. 2001;21(6):456-466.

14. Hill KM, Amir Z, Muers MF, Connolly CK, Round CE. Do newly diagnosed lung cancer patients feel their concerns are being met? Eur $J$ Cancer Care (Engl). 2003;12(1):35-45.

15. Sanson-Fisher R, Girgis A, Boyes A, Bonevski B, Burton L, Cook P. The unmet supportive care needs of patients with cancer. Supportive Care Review Group. Cancer. 2000;88(1):226-237.

16. Spiegel D. Health caring: psychosocial support for patients with cancer. Cancer. 1994;74(Suppl 4):S1453-S1457.

17. Maguire R, Papadopoulou C, Kotronoulas G, Simpson MF, McPhelim J, Irvine L. A systematic review of supportive care needs of people living with lung cancer. Eur J Oncol Nurs. 2013;17(4):449-464. 
18. Hoffman AJ, Brintnall RA, Brown JK, et al. Virtual reality bringing a new reality to postthoracotomy lung cancer patients via a home-based exercise intervention targeting fatigue while undergoing adjuvant treatment. Cancer Nurs. 2014;37(1):23-33.

19. Chen CI, Kuan CF, Miser J, et al. Comorbidity as an independent risk factor in patients with cancer: an 8-year population-based study. Asia Pac J Public Health. Epub March 27, 2013.

20. Naylor MD, Brooten D, Campbell R, et al. Comprehensive discharge planning and home follow-up of hospitalized elders: a randomized clinical trial. JAMA. 1999;281(7):613-620.

21. Janssen-Heijnen ML, Maas HA, Houterman S, Lemmens VE, Rutten HJ, Coebergh JW. Comorbidity in older surgical cancer patients: influence on patient care and outcome. Eur J Cancer. 2007;43(15): 2179-2193.

22. Lüchtenborg M, Jakobsen E, Krasnik M, Linklater KM, Mellemgaard A, Møller H. The effect of comorbidity on stage-specific survival in resected non-small cell lung cancer patients. Eur J Cancer. 2012;48(18):3386-3395.

23. Institute of Medicine. Delivering High-Quality Cancer Care: Charting a New Course for a System In Crisis. Washington, DC: The National Academies Press; 2013.

24. Institute of Medicine. From Cancer Patient to Cancer Survivor: Lost In Transition. Washington, DC: The National Academies Press; 2006.

25. Wen K, Gustafson D. Needs assessment for cancer patients and their families. Health Qual Life Outcomes. 2004;2:11-23.

26. Morse JM, Richards L. README FIRST for a User's Guide to Qualitative Methods. Thousand Oaks, CA: Sage Publications, Inc.; 2002.

27. Krueger RA, Casey MA, editors. Focus Groups: A Practical Guide for Applied Research. 4th ed. Thousand Oaks, CA: Sage Publications, Inc.; 2009.

28. Hsieh HF, Shannon SE. Three approaches to qualitative content analysis. Qual Health Res. 2005;15(9):1277-1288.

29. Hoffman AJ, von Eye A, Gift AG, Given BA, Given CW, Rothert M Testing a theoretical model of perceived self-efficacy for cancer-related fatigue self-management and optimal physical functional status. Nurs Res. 2009;58(1):32-41.

30. Hoffman AJ. Enhancing self-efficacy for optimized patient outcomes through the theory of symptom self-management. Cancer Nurs. 2013;36(1):E16-E26.
31. Naylor MD, Brooten DA, Campbell RL, Maislin G, McCauley KM, Schwartz JS. Transitional care of older adults hospitalized with heart failure: a randomized, controlled trial. J Am Geriatr Soc. 2004;52(5): 675-684.

32. Clark K, Bardwell WA, Arsenault T, DeTeresa R, Loscalzo M. Implementing touch-screen technology to enhance recognition of distress. Psychooncology. 2009;18(8):822-830.

33. Montazeri A, Milroy R, Hole D, McEwen J, Gillis CR. Anxiety and depression in patients with lung cancer before and after diagnosis: findings from a population in Glasgow, Scotland. J Epidemiol Community Health. 1998;52(3):203-204.

34. Sarna L, Padilla G, Holmes C, Tashkin D, Brecht ML, Evangelista L. Quality of life of long-term survivors of non-small cell lung cancer. J Clin Oncol. 2002;20(13):2920-2929.

35. Liao YC, Liao WY, Shun SC, Yu CJ, Yang PC, Lai YH. Symptoms, psychological distress, and supportive care needs in lung cancer patients. Support Care Cancer. 2011;19(11):1743-1751.

36. Cleeland CS, Zhao F, Chang VT, et al. The symptom burden of cancer: Evidence for a core set of cancer-related and treatment-related symptoms from the Eastern Cooperative Oncology Group Symptom Outcomes and Practice Patterns study. Cancer. 2013;119(24):4333-4340.

37. Simoff MJ, Lally B, Slade MG, et al. Symptom management in patients with lung cancer: diagnosis and management of lung cancer. American College of Chest Physicians evidence-based clinical practice guidelines. Chest. 2013;143(Suppl 5):S455-S497.

38. National Comprehensive Cancer Network. NCCN Guidelines Version 1.2013. Survivorship. Fort Washington, PA: National Comprehensive Cancer Network; 2013. Available from: https://subscriptions.nccn.org/ gl_login.aspx?ReturnURL=http://www.nccn.org/professionals/physician_gls/pdf/survivorship.pdf. Accessed November 1, 2013.

39. Satia JA, Campbell MK, Galanko JA, James A, Carr C, Sandler RS. Longitudinal changes in lifestyle behaviors and health status in colon cancer survivors. Cancer Epidemiol Biomarkers Prev. 2004;13(6): 1022-1031.

40. Barbera L, Seow H, Howell D, et al. Symptom burden and performance status in a population-based cohort of ambulatory cancer patients. Cancer. 2010;116(24):5767-5776.

41. Li J, Girgis A. Supportive care needs: are patients with lung cancer a neglected population? Psychooncology. 2006;15(6):509-516.
Lung Cancer: Targets and Therapy

\section{Publish your work in this journal}

Lung Cancer: Targets and Therapy is an international, peer-reviewed, open access journal focusing on lung cancer research, identification of therapeutic targets and the optimal use of preventative and integrated treatment interventions to achieve improved outcomes, enhanced survival and quality of life for the cancer patient. Specific topics covered in the journalinclude: Epidem of life fo ection and screening Cellular research and biomarkers; Identification of biotargets and agents with nove

\section{Dovepress}

mechanisms of action; Optimal clinical use of existing anticancer agents, including combination therapies; Radiation and surgery; Palliative care; Patient adherence, quality of life, satisfaction; Health economic evaluations. The manuscript management system is completely online and includes a very quick and fair peer-review my system. Visit http://www.dovepress.com/testimonials.php to read real quotes from
published authors. 